\title{
Crisis social chilena y salud mental: una mirada desde el ciclo vital
}

\author{
Ana Barrera-Herrera ${ }^{1}$, María José Baeza-Rivera² ${ }^{2}$ Neli Escandón-Nagel ${ }^{3}$, \\ Jorge Constanzo ${ }^{4}$, Emilio Moya ${ }^{5}$ y Bertha Escobar ${ }^{6}$ \\ Universidad Católica de Temuco-Chile
}

Durante 2019 Chile fue protagonista de masivas manifestaciones ciudadanas motivadas por desigualdades históricas. El objetivo del estudio fue comparar la percepción de adultos emergentes y adultos chilenos sobre la protesta social y su relación con salud mental y bienestar social. 771 personas respondieron una encuesta en línea sobre las variables de estudio. Los resultados indican que los adultos sobre 30 años presentan mayor interés en la política y ambos grupos manifiestan un rechazo transversal al uso de violencia en protestas ciudadanas. Además, los adultos emergentes lideraron en anomia y afecto negativo, mientras que los adultos presentan mayor bienestar social y satisfacción con la vida. La protesta social chilena ha tenido consecuencias políticas y socioemocionales, las cuales permanecen hasta la actualidad.

Palabras clave: adultez emergente, adultez, política, crisis social, salud mental

\section{The Chilean social crisis and mental health: a glance from the life cycle}

During 2019, Chilean society witnessed massive citizen demonstrations motivated by historical inequalities. The objective of this study is to compare the perception of young adults and adults about the social protests and its relation to mental health and social well-being.

1 Doctora en Psicología. Académica del Departamento de Psicología en la Universidad Católica de Temuco, Chile. Dirección postal: Manuel Montt 56, Temuco, Chile. Correo electrónico: abarrera@uct.cl. https://orcid.org/0000-0003-0574-6568

2 Doctora en Psicología. Académica del Departamento de Psicología en la Universidad Católica de Temuco, Chile. Dirección postal: Manuel Montt 56, Temuco, Chile. Correo electrónico: maria.baeza@uct.cl. https://orcid.org/0000-0003-0574-6568

3 Doctora en Psicología Clínica y de la Salud. Académica del Departamento de Psicología en la Universidad Católica de Temuco, Chile. Dirección postal: Manuel Montt 56, Temuco, Chile. Correo electrónico: nescandon@uct.cl. https://orcid.org /0000-0002-6697-2692

4 Magíster en Planificación y Gestión Territorial. Académica del Departamento de Psicología en la Universidad Católica de Temuco, Chile. Dirección postal: Manuel Montt 56, Temuco, Chile. Correo electrónico: dago.constanzo@gmail.com. https://orcid.org 10000-0002-3276-3892

5 Doctor en Estudios Latinoamericanos. Académica del Departamento de Psicología en la Universidad Católica de Temuco, Chile. Dirección postal: Manuel Montt 56, Temuco, Chile. Correo electrónico: emoya@uct.cl. https://orcid.org /0000-0001-9659-4170

6 Magíster en Salud Pública. Decana de la Facultad de Ciencias de la Salud en la Universidad Católica de Temuco, Chile. Dirección postal: Manuel Montt 56, Temuco, Chile. Correo electrónico: bescobar@uct.cl, https://orcid.org /0000-0001-5768-1845 
711 people answered the online survey on the study factors. The results indicate that adults above the age of 30 show more interest in politics and both groups categorically reject the use of violence during the civilian protests. Moreover, the young adults showed higher results in anomie and negative affectivity, whereas adults show better social well-being and satisfaction with live. The Chilean social protest has had political and socio-emotional consequences, which remain until today.

Keywords: emerging adulthood, adulthood, politics, social crisis, mental health

\section{Crise social chilena e saúde mental: um olhar desde o ciclo de vida}

Durante 2019, o Chile foi protagonista de massivas manifestaçōes cidadãs motivadas por desigualdades históricas. O objetivo deste estudo foi o de comparar a percepção dos adultos emergentes e adultos chilenos sobre o protesto social e sua relação com a saúde mental e bem-estar social. Um questionário on-line sobre as variáveis do estudo foi respondido por 771 pessoas. Os resultados indicaram que os adultos acima de 30 anos apresentam maior interesse em política e, ambos os grupos, manifestam rejeição transversal ao uso da violência em protestos cidadãos. Os adultos emergentes apresentaram maior anomia e afeto negativo, enquanto os adultos apresentaram maior bem-estar social e satisfaçáo com a vida. O protesto social chileno teve consequências políticas e socioemocionais que permanecem até hoje. Palavras-chave: idade adulta emergente, idade adulta, política, crise social, saúde mental.

\section{La crise sociale chilienne et la santé mentale: un aperçu du cycle de vie}

En 2019, le Chili fut au premier plan de manifestations citoyennes massives motivées par les inégalités historiques. L'objectif de cette étude est de comparer la perception des jeunes adultes et des adultes chiliens sur la contestation sociale et leurs relations avec la santé mentale et bien-être. Un échantillon de 771 personnes a répondu à une enquête en ligne sur les variables analysées dans cette étude. Les résultats indiquent que les adultes de plus de 30 ans présentent un plus grand intérêt pour la politique et que les deux groupes expriment un rejet catégorique au recours à la violence pendant les manifestations citoyennes. Également, les jeunes adultes présentent des indices d'anomie et d'affectivité négative tandis que les adultes présentent un meilleur bien-être social et satisfaction dans la vie. La contestation sociale chilienne a eu des conséquences politiques et socio-émotionnelles, lesquelles persistent jusqu'au jour d'aujourd'hui.

Mots-clés: âge adulte émergent, l'âge adulte, politique, crise sociale, santé mentale. 
En octubre del año 2019, Chile fue protagonista de una protesta social masiva propiciada por el descontento con la situación actual del país y las escasas respuestas gubernamentales frente a diversas demandas sociales en torno a temas como el transporte, la educación, la salud, el sistema previsional, vivienda, el medio ambiente, la corrupción, entre otros. Este estallido social ha sido un fenómeno compartido a nivel latinoamericano, donde diversos países, como Chile, Ecuador, Colombia, Perú, Guatemala, entre otros, han puesto en evidencia un malestar creciente entre la población, motivado por un conjunto de desigualdades y problemas sociales no resueltos, los cuales erosionan el lazo social y cuestionan la legitimidad de las instituciones en el país (Ortega, 2017; Reyes; 2019; Vera, 2017). Debido a la transversalidad de las demandas y a una búsqueda compartida por mejoras de las condiciones sociales y de salud tanto física como mental de la población, esta crisis en Chile ha desencadenado diversas movilizaciones, las que han convocado a miles de personas a las calles, tanto a jóvenes como adultos.

Los jóvenes chilenos son un grupo que se ha caracterizado por tener un rol protagónico en la protesta social, cuyo afán ha sido visibilizar una serie de legítimas demandas que abogan por mejoras en salud, pensiones, trabajo e incluso la esfera constitucional (Instituto Nacional de la Juventud [INJUV], 2018). Actualmente, en Chile existen más de cuatro millones de jóvenes entre 18 y 29 ańos (Instituto Nacional de Estadística [INE], 2017), los cuales transitan por un periodo de vida llamado adultez emergente, el cual se caracteriza por ser una fase de transición entre la adolescencia y la adultez, un camino complejo que tiene por propósito fundamental prepararse para ser adulto, y un periodo que presenta una llamativa dualidad (Arnett, 2000; 2004; 2008). Por una parte, es una etapa de gran optimismo y posibilidades, pues existen mayores niveles de independencia, sin grandes responsabilidades con otros, el futuro permanece abierto y pocos aspectos de 
la vida han sido definidos de modo determinante. Así lo corrobora el Instituto Nacional de la Juventud, que señala que un $84.2 \%$ de los jóvenes declara estar feliz o muy feliz, y la gran mayoría cree que tendrá un mejor futuro que el que tuvieron sus padres (INJUV, 2018).

Sin embargo, por otro lado, es una etapa de gran inestabilidad: el plan de vida está sujeto a numerosas revisiones y pueden existir diversos cambios, en el hogar, trabajo, carrera o en el amor, y también en la salud mental (Arnett, 2008). Las investigaciones señalan que, en esta etapa de vida, es donde la mayoría de los trastornos mentales tiene su inicio, presentándose altas prevalencias de trastornos como depresión, ansiedad, estrés, trastornos adaptativos, trastornos de la conducta alimentaria, consumo de sustancias y riesgo suicida (Arrieta et al., 2014; Baader et al., 2014; Micin y Bagladi, 2011; Ministerio de Salud [MINSAL], 2018; Stallman y Shochet, 2009).

Esta misma dualidad se puede observar cuando se analiza la presencia de los jóvenes en el ámbito político-social. Por una parte, los jóvenes chilenos han sido protagonistas activos de diversos movimientos sociales y estudiantiles, los cuales han resaltado demandas, tales como financiamiento, acceso y educación de calidad en la Educación Superior, democracia participativa, derechos humanos, justicia social, entre otros tópicos relevantes (López y Prado, 2016). No obstante, si se analizan las cifras relacionadas al interés y participación política de este grupo etario, su desafección es marcada respecto a otros grupos de edad; según la $9^{a}$ Encuesta Nacional de Juventud, un $82.5 \%$ de los jóvenes está poco o nada interesado en política (INJUV, 2018), alrededor del 1.2\% participa de algún partido político, y más del $60 \%$ de jóvenes no sufragó en la última elección presidencial del año 2017 (Servicio Electoral de Chile [SERVEL], 2017).

¿Cómo explicar esta baja participación política? Según lo que señalan algunos estudios, los jóvenes presentan un marcado desinterés por las prácticas políticas actuales, las cuales no abordan, según su percepción, valores como la solidaridad, la equidad y la tolerancia (Corporación PARTICIPA, 2003). Sin embargo, esta estructura tradicional de participación ha permitido que los jóvenes desarrollen otras 
formas de participación alternativas, que constituyen también expresiones democráticas, tales como las movilizaciones, y el planteamiento de demandas y trabajo conjunto con otros sectores de la sociedad (Virgili et al., 2015).

Por su parte, los adultos chilenos sobre 30 años, son un grupo que ha sido protagonista de la transición de un régimen autoritario como fue la Dictadura militar, a un régimen político de tipo democrático. Sin embargo, durante el proceso de transición se percibe un creciente desajuste entre una sociedad que ha experimentado positivos cambios económicos y sociales, no obstante, los liderazgos políticos son percibidos como una entidad cada vez más distante y poco representativa de la población (Valenzuela, 2011).

Lo anterior se refleja en el bajo interés en la política: un 83\% de los adultos chilenos se declara poco o nada interesado en la política, siendo transversal esta afección tanto en jóvenes como en adultos; además, el grupo de adultos se muestra más insatisfecho con la democracia que la población juvenil (INJUV, 2018). Sin embargo, al analizar la participación política en las últimas elecciones el escenario es distinto: un $63.3 \%$ de los adultos reporta haber concurrido a votar en las elecciones presidenciales del año 2017 (INJUV, 2018), porcentaje más alto en comparación al de los jóvenes chilenos.

Desde un punto de vista evolutivo, según Erikson (1985, citado en Zacarés y Serra, 2011) las personas cercanas a los 40 años se dirigen hacia la séptima crisis normativa denominada "generatividad versus estancamiento". La generatividad consiste en la preocupación por encaminar y guiar a la generación siguiente y perpetuarse a sí mismos, a través de la influencia o su legado en otros (Majón-Valpuesta et al., 2016); por su parte, el estancamiento se produce cuando prevalece un sentido de inactividad o inutilidad. Un funcionamiento psicológico saludable en la edad adulta depende entonces de la resolución de la crisis de generatividad frente a estancamiento, propiciada por un contexto de relaciones sociales y participación en distintos papeles, como trabajador, cónyuge, padre o madre, formador, y en este caso, como un ciudadano (Zacarés y Serra, 2011). 
Este ajuste psicológico o salud mental implica un sentido de bienestar psicológico, en el cual las personas sanas psicológicamente tienen actitudes positivas hacia los demás y hacia sí mismos, toman sus propias decisiones, regulan su comportamiento, propician ambientes compatibles con sus necesidades, poseen metas que dan sentido a su proyecto de vida y se esfuerzan por explorar y desarrollarse (Ryff, 1995). No obstante, este ideal puede verse modulado por ciertos determinantes psicosociales, tales como el género, la edad, el nivel socioeconómico, la percepción de seguridad, entre otros factores (Silva et al., 2016).

$\mathrm{Al}$ analizar las prevalencias sobre salud mental y acceso a la salud en el grupo de adultos, los resultados son desalentadores: un $6.2 \%$ de la población chilena cumple los criterios diagnósticos para una depresión, siendo mayor la prevalencia para el grupo de adultos sobre 45 años (Minsal, 2018). De este porcentaje, solo un 2\% de los adultos consultados se encontraba en tratamiento, y un $18.6 \%$ reporta haber recibido alguna vez tratamiento médico por problemas emocionales o consumo de drogas (Minsal, 2018). Es por ello, que una de las principales demandas asociadas a la reciente protesta social ha sido el acceso y calidad de los servicios de salud en general y la salud mental en particular, poniendo en la discusión elementos como los tiempos de espera, la atención oportuna y el acceso igualitario a tratamiento, en pos de disminuir la inequidad generada por la existencia de dos subsistemas de salud, uno público y otro privado (Fuentes-García, 2019).

Teniendo en cuenta las distinciones evolutivas y psicosociales entre estas dos etapas de vida, se hace necesario preguntarse cómo los jóvenes y los adultos afrontan y actúan frente a una crisis social que demanda un rol participativo de todos los ciudadanos. El objetivo general de la investigación fue comparar la percepción de jóvenes y adultos residentes en Chile sobre la protesta social y su relación con aspectos de la salud mental y bienestar social, según grupo etario. 


\section{Método}

\section{Participantes}

La muestra estuvo compuesta por 771 adultos residentes en Chile, seleccionados mediante un muestreo no probabilístico por conveniencia. Esta modalidad reúne a aquellas personas que deciden participar del estudio pero que no fueron seleccionados en particular por el investigador como parte de una muestra probabilística aleatorizada (Jamsen y Corley, 2007). El rango de edad de los participantes fue de 18 a 74 años; al agrupar los participantes según rango etario, un $45.3 \%$ tiene entre 18 y 29 ańos y un $54.7 \%$ entre 30 y 50 o más ańos. Respecto del sexo de los participantes, un $71.9 \%$ de la muestra eran mujeres y un $28.1 \%$ hombres.

\section{Instrumentos}

Se aplicó una encuesta en línea que incluía diversas preguntas sociodemográficas para caracterizar la muestra (edad, sexo, nivel socioeconómico autoadscrito, entre otros) y preguntas respecto la percepción individual de la movilización social, interés político, medidas del gobierno frente al movimiento social, y grado de participación en la misma. Además, para medir la salud mental y bienestar social, se aplicaron las siguientes escalas:

Escala de Anomia (Srole, 1956; adaptada de la versión de Rodríguez-García, 2006): Esta escala está compuesta por nueve ítems que miden las actitudes y creencias respecto a las percepciones de desintegración o desorganización de la sociedad. Esta escala tiene un formato de respuesta tipo Likert con 4 puntos, en el cual 1 es "muy en desacuerdo" y 4 "muy de acuerdo". El índice de confiabilidad de Cronbach en este estudio fue de 0.67.

Escala de Bienestar Social (Keyes, 1998; versión de Blanco y Díaz, 2005): Compuesta por 25 reactivos que miden la valoración que se realiza respecto a las condiciones y el funcionamiento de la sociedad a partir de cinco dimensiones: integración social, aceptación social, 
contribución social, actualización social y coherencia social. El formato de respuesta es tipo Likert con 5 opciones desde "totalmente en desacuerdo" a "totalmente de acuerdo". Existe evidencia de su uso en Chile con adecuados indicadores de confiabilidad (Cárdenas et al., 2012). Los índices de confiabilidad obtenidos en este estudio para cada dimensión son adecuados, a saber: integración social $\alpha=0.78$, aceptación social $\alpha=0.87$, contribución social $\alpha=0.79$, actualización social $\alpha=0.66$ y coherencia social $\alpha=0.61$.

Escala de Afecto Positivo y Negativo (PANAS; Watson et al., 1988; versión de Dufey y Fernández, 2012): Este instrumento mide afecto positivo y afecto negativo. El primero corresponde a emociones agradables, las que suelen asociarse al adecuado afrontamiento de conflictos; por su parte, el afecto negativo corresponde a aquellas emociones que se viven con desagrado y se vinculan frecuentemente con estrés, problemas de salud y falta de estrategias de afrontamiento a los problemas. El formato de respuesta es de tipo Likert de 5 puntos, en el cual los participantes deben indicar cuánto ha experimentado el afecto mencionado, desde "muy levemente" hasta "extremadamente". En este estudio los indicadores de consistencia interna para los dos factores de la escala son adecuados: afecto positivo $\alpha=.87$, afecto negativo $\alpha=.85$.

Escala de Satisfacción con la Vida (Diener et al., 1985): Evalúa la satisfacción global que tienen las personas respecto a su propia vida, comparando sus logros con las expectativas. Está compuesta por cinco ítems con formato de respuesta tipo Likert que originalmente tiene siete puntos, sin embargo, para efectos de la realidad cultural chilena se optó por un formato de respuesta de cinco puntos, los que van desde "muy en desacuerdo" a "muy de acuerdo", donde a mayor puntaje mayor satisfacción con la vida. Existe evidencia de su uso en Chile (Schnettler et al., 2012; Schnettler et al., 2013; Schnettler et al., 2014; Vera-Villarroel et al., 2012). La consistencia interna de la escala para esta muestra fue de .87 . 


\section{Procedimiento y consideraciones éticas}

Los participantes contestaron un instrumento en línea entre los días 22 y 24 de octubre del ańo 2018, por medio de plataforma google forms, el cual fue difundido principalmente por redes sociales (Facebook y Twitter). El formato de encuesta por internet fue escogido puesto que presenta como ventaja la posibilidad de acceder a un amplio número de personas a bajo costo y gran rapidez, disminuyendo el número de respuestas deseables socialmente (Díaz de Rada, 2012). Esta menor deseabilidad social genera estimaciones más precisas en encuestas políticas y en temas delicados (Duffy, et al., 2005) tales como los que se abordan en este artículo.

Respecto de las consideraciones éticas, el equipo de investigadores elaboró un Consentimiento informado, basado en las normas y criterios aceptados por la comunidad científica (Declaración de Singapur, Declaración de Helsinki, el Código de Nüremberg y el Código de Ética de la American Psychological Association), y los Estándares del Comité de Ética en la Investigación UC Temuco, protegiendo el bienestar de las personas, voluntariedad de la participación, el anonimato y confidencialidad. Este documento se incluyó como parte inicial de la encuesta en línea, detallando los objetivos del estudio, procedimiento, y datos de contacto de los investigadores responsables. Además, indicaba que, como forma de retribución, quien estuviese interesado/a podía proporcionar su correo electrónico para recibir los resultados preliminares más relevantes del estudio. Quienes estuvieron de acuerdo en participar indicaban su conformidad en la opción "Aceptar" y continuaban con la encuesta en dicha plataforma. Cabe mencionar que las personas podían abandonar la encuesta en cualquier momento.

\section{Análisis estadístico}

Por medio del software estadístico SPSS versión 26.0 primeramente se realizaron análisis descriptivos para caracterizar la muestra y análisis de confiabilidad para las diferentes escalas incluidas en el estudio. Posteriormente, para responder a los objetivos de investigación, se realizó 
una comparación de las variables relacionadas con aspectos de participación política y percepción de la protesta social entre los dos grupos etarios por medio de la prueba Chi cuadrado. Luego, para contrastar el comportamiento de cada grupo respecto a las variables de salud mental (anomia, bienestar social, afecto positivo y negativo, y satisfacción con la vida), se realizaron prueba t de muestras independientes, considerando ambos grupos etarios.

\section{Resultados}

Primeramente, respecto de variables relacionadas al aspecto político, esto es, interés por la política, adscripción a partido político, uso de la violencia por parte de manifestantes como de policías, y participación en las manifestaciones (redes sociales y cacerolazos), los resultados dan cuenta de que existen diferencias en la mayoría de las variables: en general, los adultos sobre 30 años presentan mayor interés por la política que los adultos emergentes $\left(\chi^{2}=25.281, p<.001\right)$, además son quienes se identifican en su mayoría con partidos de izquierda, mientras que la mayor parte de los adultos emergentes no se identifica con ningún partido político $\left(\chi^{2}=16.371, p<.001\right)$. En ambos grupos se presenta un bajo acuerdo con el uso de la violencia por parte de los manifestantes, aunque en adultos el rechazo es aún mayor $\left(\chi^{2}=10.262\right.$, $p<.01)$. Además, en ambos grupos también se observa participación activa en cuanto a manifestar su descontento en redes sociales, siendo los jóvenes quienes más utilizan esta vía de expresión $\left(\chi^{2}=5.784\right.$, $p<.01)$. Los resultados se observan en la Tabla 1. 
Crisis social chilena y salud mental: una mirada desde el ciclo vital / Barrera-Herrera et al.

\section{Tabla 1}

Comparación en variables de participación politica entre adultos emergentes $y$ adultos.

\begin{tabular}{|c|c|c|c|c|}
\hline Variable & Adultos emergentes & Adultos & $\chi^{2}$ & $p$ \\
\hline \multicolumn{5}{|l|}{ Interés por la política } \\
\hline Bajo & $22.3 \%$ & $14.2 \%$ & 25.281 & $<.001$ \\
\hline Medio & $34.1 \%$ & $24.2 \%$ & & \\
\hline Alto & $43.6 \%$ & $61.6 \%$ & & \\
\hline Identificación política & & & 16.371 & $<.001$ \\
\hline Derecha & $14.6 \%$ & $17 \%$ & & \\
\hline Izquierda & $34.1 \%$ & $46 \%$ & & \\
\hline Ninguna & $51.3 \%$ & $37 \%$ & & \\
\hline $\begin{array}{l}\text { Acuerdo con el uso de } \\
\text { la violencia por parte de } \\
\text { manifestantes }\end{array}$ & & & 10.262 & $<.01$ \\
\hline Bajo & $71.3 \%$ & $80.6 \%$ & & \\
\hline Medio & $16.9 \%$ & $13 \%$ & & \\
\hline Alto & $11.7 \%$ & $6.4 \%$ & & \\
\hline $\begin{array}{l}\text { Acuerdo con el uso de la } \\
\text { violencia por parte de policías }\end{array}$ & & & 3.054 & .217 \\
\hline Bajo & $89.1 \%$ & $84.6 \%$ & & \\
\hline Medio & $6 \%$ & $8.5 \%$ & & \\
\hline Alto & $4.9 \%$ & $6.6 \%$ & & \\
\hline $\begin{array}{l}\text { Participación en las } \\
\text { manifestaciones en calles }\end{array}$ & & & 2.258 & .133 \\
\hline Sí & $70.5 \%$ & $65.4 \%$ & & \\
\hline No & $29.5 \%$ & $34.6 \%$ & & \\
\hline Participación en redes sociales & & & 5.784 & $<.01$ \\
\hline Sí & $84 \%$ & $77 \%$ & & \\
\hline No & $16 \%$ & $33 \%$ & & \\
\hline
\end{tabular}


En segundo lugar, con relación a las variables asociadas a salud mental y bienestar social, los resultados dan cuenta de que existen diferencias estadísticamente significativas entre ambos grupos en la mayoría de las variables, a saber: anomia $(t=5.53, p<.001)$, afecto negativo $(t=$ $4.38, p<.001)$, integración social $(t=-5.76, p<.001)$, aceptación social $(t=-3.36, p<.01)$, actualización social $(t=-3.16, p<.01)$, coherencia social $(t=-2.52, p<.05)$, contribución social $(t=-5.18, p<.001)$, y satisfacción con la vida $(t=-5.67, p<.001)$. En general, los tamaños de efectos son pequeños a medianos (ver Tabla 2).

\section{Tabla 2}

Diferencia de medias entre adultos emergentes y adultos en salud mental $y$ bienestar social.

\begin{tabular}{lcccccc}
\hline Variable & $\begin{array}{c}\text { Promedio } \\
\text { adultos } \\
\text { emergentes }\end{array}$ & $\begin{array}{c}\text { Promedio } \\
\text { adultos }\end{array}$ & $t$ & $p$ & $\begin{array}{c}\text { Tamaño } \\
\text { del efecto } \\
d \text { Cohen }\end{array}$ \\
\hline Anomia & $2.68(.45)$ & $2.50(.46)$ & 5.53 & $<.001$ & .39 \\
Afecto positivo & $3.49(.85)$ & $3.41(.77)$ & 1.37 & .169 & .09 \\
Afecto negativo & $3.02(.79)$ & $2.77(.76)$ & 4.38 & $<.001$ & .32 \\
Integración social & $3.48(.80)$ & $3.78(.65)$ & -5.76 & $<.001$ & -.41 \\
Aceptación social & $3.03(.87)$ & $3.24(.82)$ & -3.36 & $<.01$ & -.24 \\
Actualización social & $3.29(.72)$ & $3.46(.76)$ & -3.16 & $<.01$ & -.22 \\
Coherencia social & $3.92(.69)$ & $4.05(.73)$ & -2.52 & $<.05$ & -.18 \\
Contribución social & $3.99(.78)$ & $4.26(.63)$ & -5.18 & $<.001$ & -.38 \\
Satisfacción con la vida & $2.90(.91)$ & $3.27(.85)$ & -5.67 & $<.001$ & -.42 \\
\hline
\end{tabular}

Aquellas variables en que los adultos emergentes presentan mayores promedios son: anomia y afecto negativo, mientras que en las variables restantes (integración social, aceptación social, actualización social, coherencia social, contribución social y satisfacción con la vida), el promedio de los adultos sobre 30 ańos es mayor que el de los adultos emergentes. Es decir, los adultos presentan mayor sensación de que pueden contribuir a la sociedad (ver Tabla 2). 


\section{Discusión}

Desde octubre del 2019, Chile ha sido protagonista de un movimiento histórico de protesta social, motivado por el descontento generalizado ante diversas problemáticas sociales, el cual ha demandado una participación activa de toda la población y que ha generado distintos comportamientos, percepciones y efectos en la salud mental en sus protagonistas. Desde esta perspectiva, si bien tanto adultos emergentes como adultos sobre 30 ańos cuentan con distintas trayectorias de vida, los resultados de este estudio revelan que ambos grupos muestran un renovado interés por la política, siendo más elevado este en el grupo de adultos sobre 30 ańos. Lo anterior refleja que la protesta social ha sido un evento que ha generado un interés masivo en la población, y que las demandas contenidas en el estallido social han despertado un interés y preocupación generalizada, independiente de la etapa de vida en que se encuentre el ciudadano chileno. Aun cuando el grupo de adultos denota un mayor interés político, lo que se condice con estudios previos donde existe evidencia de una participación más activa en eventos como elecciones (SERVEL, 2017), la brecha actual entre jóvenes y adultos no es tan amplia si se compara con los datos previos al inicio de la protesta social.

Otro punto en que coinciden ambos grupos etarios corresponde al rechazo transversal al uso de violencia por parte de manifestantes y policías en las protestas ciudadanas que caracterizaron este movimiento social. Tanto jóvenes como adultos rechazan escenarios de violencia social que han llevado a algunos manifestantes a dañar espacios públicos o el uso indiscriminado de medidas de represión y control social, como bombas lacrimógenas, uso de armas y violencia física por parte de algunos policías. Si bien ambos grupos rechazan la violencia ejercida por manifestantes y policías, es llamativo que los adultos sobre 30 años rechacen de forma más tajante la violencia en manifestaciones sociales, en comparación con los adultos emergentes. Este rechazo a la violencia puede estar relacionado directamente a los procesos históricos de Chile, caracterizados por desigualdades e injusticias, además de las 
consecuencias psicosociales y políticas relacionadas a la violación de los derechos humanos durante la dictadura militar (Madariaga, 2019). Probablemente este doloroso pasado ha permitido que hoy en día los chilenos, sobre todo quienes vivieron el periodo de la dictadura, rechacen estas acciones de violencia y aboguen por un nuevo pacto social, de carácter democrático, que tenga en cuenta como valor primordial el respeto a los derechos humanos individuales y colectivos. Por su parte, los adultos emergentes, si bien rechazan la violencia, generacionalmente en los últimos años han liderado distintas manifestaciones sociales (especialmente manifestaciones universitarias), donde probablemente ciertos actos de violencia reactiva tales como marchas, tomas, huelgas, o incluso actos considerados delictuales pueden ser considerados como formas de expresión frente a la frustración, anomia y represión policial (Acevedo et al., 2019; INJUV, 2018; Madariaga, 2019).

En relación a la identificación política actual de jóvenes y adultos, este estudio revela que existen diferencias importantes: mientras que más de la mitad de los adultos emergentes no se adscriben a ningún partido político, la mayor parte de los adultos se autodefine como de izquierda. Este resultado se condice con los resultados de diversos estudios: por ejemplo, la $9^{\text {a }}$ Encuesta nacional de la Juventud refleja que un $64.5 \%$ de los jóvenes chilenos encuestados no se identifica con ningún sector político, aun cuando un $54.7 \%$ piensa que la democracia es preferible a cualquier otra forma de gobierno (INJUV, 2018). Si bien la mayor parte de los jóvenes no adhiere a un partido político o no participa tan activamente en procesos eleccionarios debido a sus críticas en cuanto al funcionamiento de la política chilena, distancia con instituciones y personajes políticos en general (González et al., 2005; Navarrete, 2008), este grupo prefiere otro tipo de participación democrática, como por ejemplo las organizaciones sociales, o manifestaciones a través de marchas o redes sociales. Esto último coincide con los resultados de este estudio, en que se aprecia que, si bien ambos grupos de comparación utilizan las redes sociales como vía de manifestación, son los adultos emergentes quienes más optan por su uso (INJUV 2018; Scherman et al., 2013; Vargas-Salfate et al., 2015). Esta nueva forma 
de participación social demanda una actualización y renovación de la cultura política dominante, hacia formas de participación social más efectivas e inclusivas con los diferentes grupos de la población.

Respecto a la salud mental y bienestar social, los resultados comparativos entre ambos grupos son preocupantes y reveladores. Por una parte, los adultos emergentes chilenos muestran resultados más elevados en las variables de anomia y afecto negativo. Los jóvenes chilenos se han identificado mayormente con reactivos como "Los políticos no están interesados en lo que les pasa a los chilenos promedio", o "En estos días una persona no sabe realmente con quien puede contar", revelando una percepción de una sociedad desintegrada, una relación débil de sí mismos con el mundo social del que forman parte, específicamente respecto a la percepción sobre el orden provisto por las normas que organizan su experiencia como individuos (Aceituno et al., 2009; Srole, 1956). Probablemente esta falta de orden o integración pueda estar vinculada a las demandas y frustraciones de los jóvenes con las instituciones políticas y con la falta de integración a espacios de participación política que no responden a sus intereses y formas de participación preferentes, factores que han derivado en un marcado desinterés en este ámbito o más bien un desinterés con las formas habituales de participación política.

Asimismo, este estado de anomia en los jóvenes puede verse vinculado a las expresiones de afecto negativo, que pueden estar presentes previo al estallido social e intensificarse por este proceso histórico. Emociones como molestia, nervios, temor, intranquilidad, entre otras, se vinculan con estados emocionales negativos que integran los trastornos de salud mental más frecuentes en esta población (Arrieta et al., 2014; Baader et al., 2014; Barrera-Herrera et al., 2019; Micin y Bagladi, 2011; Stallman y Shochet, 2009), por tanto, esta crisis social agrega una nueva carga de efectos negativos sobre la salud mental (Madariaga, 2019), no solo para los jóvenes chilenos, sino para toda la población.

Esta crisis responde a un estado de desasosiego emocional acumulado por décadas, un estado de malestar manifestado en la alta prevalencia de diferentes problemas de salud mental, una demanda de 
atención en salud mental insuficientemente atendida, la existencia de un alto consumo de psicofármacos y el aumento progresivo de licencias médicas, problemas que deben de ser tenidos en consideración, pues evidencian graves dificultades de salud pública vigentes, que podrían estar estrechamente vinculadas con el malestar social (Aceituno et al., 2012).

Un resultado más alentador corresponde a que los adultos de esta muestra presentan puntajes más altos en todas las dimensiones que componen el bienestar social, además de un mayor grado de satisfacción con la vida. Lo anterior demuestra un grado de valoración adecuado respecto a las condiciones y el funcionamiento de la sociedad (Keyes, 1998), y revela que, aun cuando la historia política de Chile cuenta con episodios que han mermado la confianza en los demás y, consecuentemente, pudieran implicar un menor involucramiento con la vida en comunidad, afectando aspectos como la integración social, el grupo de adultos sobre 30 años de esta muestra da señales de un proceso de resiliencia social, que refleja un cierto grado de confianza en el desarrollo de la sociedad y un intento por establecer un pacto social compartido que recoja el diálogo, el respeto y la inclusión como aspectos indiscutibles para el logro del bienestar colectivo (Soto-Salcedo y Andrade-Pérez, 2019). Probablemente por la etapa de vida en la que se encuentran y por los desafíos vitales asociados (generatividad versus estancamiento) los adultos posean una preocupación y un anhelo por la posibilidad de un cambio social, que represente sus ideales, pero que al mismo tiempo ceda el protagonismo al ímpetu de las generaciones más jóvenes en la búsqueda de un bienestar colectivo.

\section{Conclusiones}

Este estudio presenta un panorama descriptivo de cómo una protesta social de carácter nacional impacta de forma diferente según la etapa vital en que transiten sus protagonistas, cumpliéndose el objetivo primario de comparar la percepción de jóvenes y adultos residentes en 
Chile sobre la protesta social y su relación con aspectos de la salud mental y bienestar social. Esta aproximación logra esclarecer similitudes y semejanzas que existen en ambos grupos, respecto al rechazo a la violencia, un renovado interés por la política debido a las protestas sociales, además de poner el foco en brechas generacionales ligadas a identificación política y al impacto emocional de este estallido social, en áreas como anomia, afectividad, bienestar y satisfacción con la vida.

Esta investigación también presenta ciertas limitaciones. Una de ellas, es que se utilizó un muestreo no probabilístico, el cual contempla una muestra de voluntarios, autoseleccionada, por tanto, los resultados de este estudio solo son representativos de esta muestra, no pudiendo ser generalizados a la población general. No obstante, es importate señalar que esta investigación permitió tomar una primera fotografía al incipiente estallido social, recabando impresiones y percepciones de un número importante de chilenos en la primera semana de movilizaciones. Futuros estudios podrían complementar los hallazgos de esta investigación y detallar los efectos y desafíos de este proceso histórico, considerando su evolución actual.

Finalmente, considerando los antecedentes anteriormente expuestos, la protesta social del último año, ha tenido efectos movilizadores y un impacto para cada chileno, teniendo consecuencias no solo en el ámbito político, sino también un impacto socioemocional, con ciertos matices dependiendo de la edad desde donde se fije la mirada. Esta crisis social replantea un nuevo sentido de protesta social, un cuestionamiento del modelo económico, un estado de movilización desafiante para ciudadanos e instituciones, un desafío para las ciencias sociales aplicadas y un fenómeno que con sus complejas características puede ser conducente a una nueva fase de desarrollo social para todos los chilenos. 


\section{Referencias}

Aceituno, R., Asún, R., Ruiz, S., Venegas, J. I., Corbalán, F., y Reinoso, A. (2009). Anomia y alienación en estudiantes secundarios de Santiago de Chile: Resultados iniciales de un estudio comparativo 1989-2007. Psykhé, 18(2), 3-18. http://dx.doi.org/10.4067/ S0718-22282009000200001

Aceituno, R., Miranda, G., y Jiménez, A. (2012). Experiencias del desasosiego: salud mental y malestar en Chile. Revista Anales, 3, 87-102. http://dx.doi.org/10.5354/0717-8883.2012.21730

Acevedo Tarazona, Á., Correa Lugos, A., y Mejía Jerez, A. (2019). Represión estatal como dispositivo de control psicosocial en las universidades colombianas, 1981-1991. Revista Latinoamericana de Psicología, 51(2), 66-73. http://dx.doi.org/10.14349/ rlp.2019.v51.n2.2

Arnett, J. (2000). Emerging adulthood: A theory of development from the late teens through the twenties. American Psychologist, 55(5), 469-480. https://doi.org/10.1037//0003-066X.55.5.469

Arnett, J. J. (2004). Emerging adulthood: the winding road from late teens through twenties. Oxford University Press.

Arnett, J. (2008). Adolescencia y adultez emergente. Un enfoque cultural. Pearson Educación.

Arrieta, K., Díaz, S., y González, F. (2014). Síntomas de depresión y ansiedad en jóvenes universitarios: prevalencia y factores relacionados. Revista Clínica de Medicina de Familia, 7(1), 14-22. https://doi.org/10.4321/S1699-695X2014000100003

Baader, T., Rojas, C., Molina, J., Gotelli, M., Alamo, C., Fierro, C., Venezian, S., y Dittus, P. (2014). Diagnóstico de la prevalencia de trastornos de la salud mental en estudiantes universitarios y los factores de riesgo emocionales asociados. Revista Chilena de Neuro-Psiquiatría, 52(3), 167-176. https://doi.org/10.4067/ S0717-92272014000300004

Barrera-Herrera, A., Neira-Cofré, M., Raipán-Gómez, P., RiquelmeLobos, P., y Escobar, B. (2019). Apoyo social percibido y 
factores sociodemográficos en relación con los síntomas de ansiedad, depresión y estrés en universitarios chilenos. Revista de Psicopatología y Psicología Clinica., 24(2), 105-115. https://doi. org/10.5944/rppc.23676

Blanco, A., y Díaz, D. (2005). El bienestar social: su concepto y medición. Psicothema, 17(4), 582-589.

Cárdenas, M., Barrientos, J., Bilbao, A., Páez, D., Gómez, F., y Asún, D. (2012). Estructura factorial de la escala de satisfacción con la vida en una muestra de estudiantes universitarios chilenos. Revista Mexicana de Psicología, 29(2), 157-164.

Corporación PARTICIPA (2003). Jóvenes sí participan pero no creen en la clase política. https://docplayer.es/12826012-Jovenes-siparticipan-pero-no-creen-en-la-clase-politica.html

Díaz de Rada, V. (2012). Ventajas e inconvenientes de la encuesta por Internet. Papers, 97(1), 193-223.

Diener, E., Emmons, R., Larsen, R., y Griffin, S. (1985). The satisfaction with life scale. Journal of Personality Assessment, 49(1), 71-75. https://doi.org/10.1207/s15327752jpa4901_13

Dufey M., y Fernández AM. (2012). Validez y confiabilidad del Positive Affect and Negative Affect Schedule (PANAS) en estudiantes universitarios chilenos. RIDEP. 2012, 34(1), 157-73.

Duffy, B., Terhanian, G., Bremer, J., y Smith, K. (2005). Comparing data from online and face-to-face surveys. International Journal of Market Research, 47, 615-639. https://doi. org/10.1177/147078530504700602

Fuentes-García, A. (2019). El clamor de las inequidades: Estallido social y salud en Chile. Revista Chilena de Salud Pública, 23(2), 93-94.

González, R., Manzi, J., Cortés, F., Torres, D., De Tezanos, P., Aldunate, N., Aravena, M. T., y Saíz, J. L. (2005). Identidad y actitudes políticas en jóvenes universitarios: el desencanto de los que no se identifican políticamente. Revista de Ciencia Politica, 25(2), 65-90. http://dx.doi.org/10.4067/ S0718-090X2005000200003 
Instituto Nacional de Estadísticas (2017). Anuario de estadisticas vitales. https://www.ine.cl/docs/defaultsource/nacimientos-matrimonios-y-defunciones/ publicaciones-y-anuarios/anuarios-de-estad\%C3\%ADsticasvitales/anuario-de-estad\%C3\%ADsticas-vitales-2017. pdf?sfvrsn=95e68aba_4

Instituto Nacional de la Juventud (2018). Novena Encuesta Nacional de la Juventud. http://www.injuv.gob.cl/storage/docs/9\%C2\%B0_ Encuesta_Nacional_de_Juventud_2018.pdf

Jamsen, J., y Corley, K. (2007). E-survey methodology. En R. Reynolds, R. Woods, J. Baker (Eds.), Handbook of research on electronic surveys and measurements (pp. 1-8). IGI Global.

Keyes, C. (1998). Social well-being. Social Psychology Quarterly, 61, 121-140. http://dx.doi.org/10.2307/2787065

López, D. A. y Prado, M. C. (2016). ¿Qué deseaban los estudiantes universitarios chilenos el año 2011? Altre Modernità, 218-233. https://doi.org/10.13130/2035-7680/7064

Madariaga, C. (2019). El "estallido social” y la salud mental de la ciudadanía: Una apreciación desde la experiencia PRAIS. Revista Chilena de Salud Pública, 23(2), 146-156.

Majón-Valpuesta, D., Ramos, P., y Pérez-Salanova, M. (2016). Claves para el análisis de la participación social en los procesos de envejecimiento de la generación baby boom. Psicoperspectivas, 15(2), 53-63. https://doi.org/10.5027/ psicoperspectivas-vol15-issue2-fulltext-833

Micin, S. y Bagladi, V. (2011). Salud mental en estudiantes universitarios: incidencia de psicopatología y antecedentes de conducta suicida en población que acude a un servicio de salud estudiantil. Terapia Psicológica, 29(1), 53-64. https://doi.org/10.4067/ S0718-48082011000100006

Ministerio de Salud, MINSAL (2018). Encuesta Nacional de Salud 2016-2017. Segunda entrega. http://www.minsal.cl/wp-content/ uploads/2018/01/2-Resultados-ENS_MINSAL_31_01_2018. pdf 
Navarrete, B. (2008). Juventud y política en los liceos municipales: El caso de Maipú. Última década, 16(28), 167-202. http:// dx.doi.org/10.4067/S0718-22362008000100009

Ortega, L. A. (2017). Is basic science used to help solve psychosocial issues in Latin America? Revista Latinoamericana de Psicologia, 49(3), 161-162. http://dx.doi.org/10.1016/j.rlp.2016.06.004

Reyes, G. E. (2019). Latinoamérica: crisis de legitimidad. https://www. researchgate.net/profile/Giovanni_Efrain_Reyes-Ortiz/publication/337364573_Latinoamerica_Crisis_de_Legitimidad/ links/5dd41cd9299bf11ec8626b3d/Latinoamerica-Crisis-deLegitimidad.pdf

Rodríguez-García, J. M. (2006). Análisis comparativo de confiabilidad $\mathrm{y}$ validez de dos escalas de anomia. Interamerican Journal of Psychology, 40(2), 193-204.

Ryff, C. D. (1995). Psychological well-being in adult life. Current Directions in Psychological Science, 4(4), 99-104. https://doi. org/10.1111/1467-8721.ep10772395

Scherman, A., Arriagada, A., y Valenzuela, S. (2013). La protesta en la era de las redes sociales: el caso chileno. Intermedios. Medios de Comunicación y Democracia en Chile, 1, 179-197.

Schnettler, B., Miranda, H., Sepúlveda, J., Denegri, M., Mora, M., y Lobos, G. (2012). Satisfaction with life and food-related life in persons of the mapuche ethnic group in southern Chile. A comparative analysis using logit and probit models. Journal of Happiness Studies, 13(2), 225-246. http://dx.doi.org/10.1007/ s10902-011-9259-5

Schnettler, B., Miranda, H., Sepúlveda, J., Mora, M., Lobos, G., y Denegri, M. (2013). Satisfacción con la vida en personas de etnia Mapuche residentes en la Región Metropolitana, Chile. Un análisis comparativo con modelos Logit y Probit. Suma Psicológica, 20(1), 69-82. http://dx.doi.org/10.14349/sumapsi2013.1374

Schnettler, B., Denegri, M., Miranda, H., Sepúlveda, J., Mora, M., y Lobos, G. (2014). Satisfaction with life and with food-related 
life in central Chile. Psicothema, 26(2), 200-206. http://dx.doi. org/10.7334/psicothema2013.13

Servicio Electoral de Chile, SERVEL (2017). Estadisticas de participación por sexo y edad. https://www.servel.cl/ estadisticas-de-participacion-por-sexo-y-edad/

Silva, M., Loureiro, A., y Cardoso, G. (2016). Social determinants of mental health: a review of the evidence. The European Journal of Psychiatry, 30(4), 259-292.

Soto-Salcedo, A. S., y Andrade-Pérez, M. A. (2019). Desafíos desde el bienestar psicológico en la construcción de un acuerdo social. Salud y Bienestar Colectivo, 3(2), 71-76.

Srole, L. (1956). Social integration and certain corollaries: An exploratory study. American sociological review, 21(6), 709-716. http:// dx.doi.org/10.2307/2088422

Stallman,H.M.yShochet,I. (2009). Prevalenceofmental health problems in Australian university health services. Australian Psychologist, 44, 122-127. https://doi.org/10.1080/00050060902733727

Valenzuela, A. (2011). Crisis de representación y reforma política en Chile. https://www.cepchile.cl/cep/site/artic/20160304/ asocfile/20160304095843/DOC_AValenzuela_Crisis-deRepresentacion-y-Reforma-Politica-en-Chile.pdf

Vargas-Salfate, S., Oyanedel, J. C., y Torres-Vallejos, J. (2015). Socialización e interés en la política en jóvenes de Chile. Revista Latinoamericana de Ciencias Sociales, Niñez y Juventud, 13(2), 781-794. http://dx.doi.org/10.11600/1692715x.13216190814 Vera, M. A. (Ed.). (2017). Malestar social y desigualdades en Chile. Ediciones Universidad Alberto Hurtado.

Vera-Villarroel, P., Urzúa, A., Pavez, P., Celis-Atenas, K., y Silva, J. (2012). Evaluation of subjective well-being: Analysis of the satisfaction with life scale in Chilean population. Universitas Psychologica, 11(3), 719-727.

Virgili, M., Ganga, F., y Figueroa, K. (2015). Gobernanza universitaria o cogobierno: el caso de la Universidad de Concepción de Chile. 
Última década, 23(42), 187-216. http://dx.doi.org/10.4067/ S0718-22362015000100009

Watson, D., Clark, L. A., y Tellegen, A. (1988). Development and validation of brief measures of positive and negative affect: the PANAS scales. Journal of Personality and Social Psychology, 54(6), 1063.

Zacarés, J. J. y Serra, E. (2011). Explorando el territorio del desarrollo adulto: la clave de la generatividad. Cultura y Dducación, 23(1), 75-88. https://doi.org/10.1174/113564011794728533

Recibido: 2020-12-09

Revisado: 2021-11-10

Aceptado: 2021-11-15 